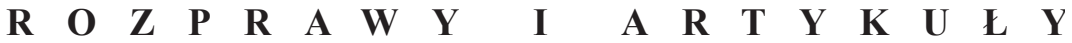

Prawo Kanoniczne

57 (2014) nr 2

\author{
COSTANTINO-M. FABRIS
}

Facoltà di Diritto Canonico "S. Pio X" di Venezia

\section{DIRITTI DEI FEDELI COME ESPRESSIONE DI VALORI}

Sommario: - 1. I diritti dei fedeli laici fino al Vaticano II. - 2. La riforma del Codice pio benedettino. - 2.1. Il dibattito pre-conciliare. - 2.2. I lavori preparatori del nuovo CIC. -3 . Il dibattito sui diritti fondamentali. -4 . Il valore persona umana alla base di obblighi e diritti dei fedeli. -5 . Alcuni fondamentali canoni alla base dei diritti di tutti i fedeli. - 6. Diritti ed obblighi dei fedeli laici.

Una delle principali caratteristiche dell'ordinamento giuridico canonico, è senza dubbio quella derivante dalla concezione antropologica, propria del cristianesimo, che vede il suo momento centrale e caratterizzante nella creazione dell'uomo ad immagine di Dio ${ }^{1}$. Questa caratteristica propria ed esclusiva dell'uomo fa sì che egli goda di una particolare dignità che trova un compiuto riconoscimento anche attraverso la garanzia di determinati diritti che di tale dignità sono la necessaria conseguenza. In quest'ottica ogni ordinamento giuridico che voglia porsi come strumento di realizzazione della giustizia, non potrà non garantire il valore e la dignità propri di ogni uomo, mediante il ri-

${ }^{1}$ Non si può non ricordare almeno il pensiero di S. Tommaso d'Aquino, il quale, partendo dalla celebre definizione di Boezio, secondo cui: «persona est rationalis naturae individua substantia» (Summa theologiae, I, q. 29, a. 1, arg. 1) si sofferma sul tema in modo assai approfondito (Summa theologiae, I, qq. 75-83), in particolare sul concetto di persona quale essere assolutamente originale ed irripetibile in quanto creata ad imago Dei; cf. R. M. PIzzorNi, I diritti fondamentali della persona umana secondo S. Tommaso d'Aquino e il magistero della Chiesa, in: F. Biffi (a cura di), I diritti fondamentali della persona umana e la libertà religiosa, Città del Vaticano 1985, 745-759. 
conoscimento di specifici diritti (con conseguenti doveri) che debbono essere riconosciuti all'uomo i virtù della sua stessa natura.

Tuttavia, soprattutto nel corso dei secoli XIX e XX, si è sviluppata una mentalità di tipo scientifico-tecnicistica le cui conseguenze si fecero inevitabilmente sentire anche nel mondo giuridico, e spesso permangono sino ad oggi²: l'uomo diviene l'unico legislatore di se stesso, dal momento che ritiene di essere in grado di darsi un sistema di leggi che garantisca un retto esercizio del diritto e che costituisca un ordine sociale giusto. Il diritto naturale, insito nella natura stessa delle cose, diviene una categoria di difficile comprensione in quanto sembra non possedere i requisiti fondamentali di certezza ed assolutezza, che solamente le leggi positive sembrano poter offrire.

La Chiesa ha dato alcune fondamentali risposte a questo mutamento di prospettiva; da un lato ha iniziato una intensa riflessione circa la adattabilità, al pensiero cristiano, della categoria dei diritti umani così come teorizzata nel pensiero laico a partire dall'Illuminismo, e ciò al fine di riscoprire il vero senso dell'uomo e della sua natura, spesso travisata da tale visione; dall'altro vi è stato un ampio dibattito che ha riguardato i diritti dei fedeli, ed in particolare la possibilità di definire dei diritti siffatti come fondamentali, attribuendogli in tal modo un valore preminente all'interno dell'ordinamento giuridico ecclesiale. Ci soffermeremo qui brevemente su quest'ultimo punto, non tanto per dar conto del dibattito che si è sviluppato sul tema in sede di revisione del CIC17, bensì per valutare quale sia il fondamento ultimo dei diritti dei fedeli e dei fedeli laici in particolare.

2 A tale mentalità non fu estraneo nemmeno il diritto ecclesiale: «el positivismo dominó, en mayor o menor medida, a la canonística postcodicial en todas sus escuelas, haciendo de la voluntad del legislador la fuente de la juridicidad, minusvalorando los derechos subjetivos y centralizando el mundo de lo juridico en torno al valor objetivo de la norma», A. De La Hera, Persona e ordinamento nel diritto sacramentale della Chiesa, in: Persona e ordinamento nella Chiesa, Milano 1975, 504; con l'espressione post-codiciale si fa qui riferimento ovviamente al CIC17. 


\section{I diritti dei fedeli laici fino al Concilio Vaticano II}

Il termine laico, in ambito ecclesiale, compare per la prima volta nel 95 d. C. nella lettera di Clemente Romano alla Chiesa di Corinto ${ }^{3}$, per distinguere le cose sacre da quelle che non lo sono. A partire da questa data, andò sempre più affermandosi una separazione tra i laici ed i fedeli che ricevevano l'Ordine sacro, per arrivare alla nota definizione di Graziano, che distingue due generi di cristiani ${ }^{4}$ : chierici e laici. La prima codificazione canonica del 1917, come noto, dal punto di vista formale manteneva una rigorosa divisione dei fedeli per status, concen-

3 J. Hervada, La definicion nominal del laico. Etimologia y uso primitivo, Ius Canonicum 8 (1968) 471-533; J. Hervada, Tres estudios sobre el uso del término laico, Pamplona 1973; P. G. CARON, I poteri giuridici del laicato nella Chiesa primitiva, Milano 1975²; J. GAUdEMET, I laici nei primi secoli della Chiesa, Communio 83-84 (1985) 12-25. Sempre sull'origine del termine laico, si veda: K. Mörsdorf, Die Stellung der Laien in der Kirche, Revue de Droit Canonique 10 (1961) 214-234.

4 «Duo sunt genera Christianorum. Est autem genus unum quod mancipatum divino offitio, et deditum contemplationi et orationi, ab omni strepitu temporalium cessare convenit, ut sunt clerici, et Deo devoti, videlicet conversi. [...] Aliud vero genus Christianorum, ut sunt laici [...] His concessum est uxorem ducere, terram colere, inter virum et virum iudicare, causas agere, oblationes super altaria ponere, decimas reddere, et ita salvari poterunt, si vicia tamen benefaciendo evitaverint», Graziano, Decretum, C. 12 , q. 1, c. 7. Come noto Graziano riprende la definizione da S. Girolamo, in particolare dalla Epistola ad quendam suum Levitam, la quale non risulta però essere tra quelle scritte dal santo. È pur vero che S. Girolamo dà una definizione di chierico, nei termini in cui ne riferisce Graziano, nella Epistola ad Nepotiarum (PL, 22, 527), ma in essa non vi è alcun riferimento ai laici. La definizione grazianea era tuttavia conosciuta sin dai tempi di S. Isidoro, il quale nell'Etymologiarum libri $X X$, forniva tanto una definizione dei chierici, quanto una definizione dei laici: «popularis; $\lambda \alpha o ́ \varsigma$ enim Grece populus dicitur» (cap. XIV, in PL 82, 294). Cf. C. S. Berardus, Gratiani canones, Taurini 1757, 197-199. Sicuramente Graziano compone a sistema definizioni già utilizzate dalla scienza canonistica, con l'intento di racchiudere in una formula schematica la più articolata realtà ecclesiale; una visione "critica" di tale impostazione si ritrova in S. Berlingò, La funzione dei laici nel nuovo C.J.C., Monitor Ecclesiasticus 107 (1982), 518-520. Sui laici nel diritto canonico medievale si vedano: H.-M. SтAмm, Die Grundrechte der Menschlichen Person im Dekret Gratians, in: I diritti fondamentali della persona umana e la libertà religiosa, 497 ss.; G. FRANSEN, Les laïcs dans l'Église au moyen âge, L'Année Canonique 29 (1985-1986) 55-69. 
trandosi in maniera quasi totale sui chierici e sui religiosi; l'individuo, il soggetto del diritto, essendo definito soprattutto in senso formale, perdeva, entro tale codificazione, i suoi aspetti sostanziali che invece, proprio a motivo delle peculiarità del diritto ecclesiale, costituiscono un elemento fondamentale per l'individuazione di tale soggetto (l'uomo) anche dal punto di vista giuridico, soprattutto avendo riguardo all" "uomo-fedele", il christifidelis $5^{5}$ della nuova codificazione 6 . In particolare la tripartizione per status ${ }^{7}$ dei fedeli, operata dal CIC del 1917 , non ponendo dal punto di vista formale tutti i battezzati sullo stesso piano, ne comportava di conseguenza anche un diverso valore sul piano sostanziale. Volendo tale tripartizione sottolineare il principio gerarchico vigente nella Chiesa e le diversità esistenti fra le varie membra del medesimo corpo, si ottenne tuttavia il risultato di incentrare gran parte delle norme sulla funzione dei chierici e dei religiosi ${ }^{8}$, restando la categoria della persona in quanto tale, solamente come concetto subalterno o, come anche sostenuto forse eccessivamente, di creare una vera "contrapposizione" tra laicato e clero". Appare piuttosto corretto

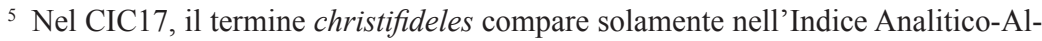
fabetico del Codice; vedi Codex Iuris Canonici anno1917 Pii X pontificis maximi iussu digestus Benedicti papae XV, Città del Vaticano 1996 (ristampa anastatica), 752.

${ }^{6}$ A tal proposito Lo Castro ricordando che, anche per il legislatore canonico del 1917, la dignità della persona umana rimaneva comunque la fonte primaria di diritti e doveri, tuttavia ammetteva che il CIC17 si era mosso: «lungo l'asse affatto opposto, espressione dell'idea della priorità del momento normativo, e dell'autorità che lo pone, rispetto alla realtà umana; idea per la quale è l'ordinamento giuridico ad attribuire personalità, soggettività, capacità giuridica, ad essere propriamente 'fonte' delle medesime», G. Lo CASTRO, La rappresentazione giuridica della condizione umana nel diritto canonico, Il Diritto Ecclesiastico 92 (1981) 242.

${ }^{7}$ L'ordinamento canonico, in questo senso, riprendeva anche il concetto di personastatus di derivazione romanistica; nel diritto romano, infatti, non bastava essere uomo per essere persona in senso giuridico, l'uomo doveva possedere ulteriori qualità legate al suo status proprio: doveva essere libero (status libertatis), doveva essere cittadino (status civitatis), non doveva essere soggetto al pater familias (nello status familiae). Vedi fra gli altri M. MARrone, Istituzioni di diritto romano, Palermo 1989, 249-374.

${ }^{8}$ I laici, tra l'altro, vedevano considerata la loro posizione solo in senso negativo: non chierici.

${ }^{9}$ P. A. Bonnet, Habet pro conditione dignitatem libertatemque filiorum Dei, Il Diritto Ecclesiastico 92 (1981) 548-620. 
affermare che, non essendovi stato un sufficiente approfondimento ecclesiologico sulla nozione di popolo di Dio, difficilmente si sarebbe potuti giungere ad un superamento del rigido dualismo chierici-laici. Il Viladrich sottolinea come, alla base di tale visione di Chiesa come societas inaequalis, vi siano sostanzialmente due fraintendimenti:

da una parte, la confusione delle nozioni di fedele e laico; e dall'altra, la traduzione della distinzione sacerdozio comune-sacerdozio ministeriale nella formula "coincidente" stato clericale-laicato, attribuendo a quest'ultima dicotomia - che si formò a partire dagli schemi mentali della società per stati dell'Ancien Régime - la natura di diritto divino che indubbiamente la prima possiede. In tale contesto, la Chiesa era per diritto divino una società disuguale, formata da sacerdoti e laici, governanti e governati, membri attivi e membri passivi, nella quale la titolarità del potere, della missione e dei fini ecclesiali era costituzionalmente attribuita a uno degli status, quello clericale ${ }^{10}$.

Tale rigida suddivisione di ruoli tra i membri della Chiesa non ha impedito autorevoli approfondimenti da parte di Autori che hanno sottolineato l'importanza dei fedeli laici per la vita della comunità ecclesiale. Non si può non ricordare almeno le fondamentali tesi del Card. Newman circa il ruolo dei fedeli nella salvaguardia del dogma cattolico ${ }^{11}$.

${ }^{10}$ P.-J. VILADRICH, La distinzione essenziale sacerdozio comune-sacerdozio ministeriale e i principi di uguaglianza e di diversità nel diritto costituzionale canonico moderno, Il Diritto Ecclesiastico 83 (1972) 124.

${ }^{11}$ J. H. Newman, Sulla consultazione dei fedeli in materia di dottrina, Brescia 1991. L'opera fu pubblicata per la prima volta col titolo On Consulting the Faithful in Matters of Doctrine, nel 1859, sulla rivista The Ramblers, e poi ristampata col medesimo titolo, ma con alcune aggiunte, nel 1871 come appendice alla terza edizione dell'opera Gli ariani nel IV secolo. Come è stato autorevolmente affermato, riprendendo le tesi del Card. Newman: «Il sensus fidei, che si traduce nel consensus fidelium, finisce con l'essere la coscienza collettiva della Chiesa. È il volto nuovo del consenso e della partecipazione corresponsabile dei fedeli alla vita di comunione; è il criterio finale, la meta ultima e la forma ideale di vita nell'ordinamento ecclesiale. [...] La recezione della fede e delle norme che la traducono nella vita quotidiana dell'ordinamento è conferma di verità, è un'altra forma della comunione ecclesiale; capace di creare una sostanziale parità fra laici e chierici, di porre entrambi in modo uguale sotto l'unica Parola del Signore, il carisma del sensus fidei appare il "momento genetico comune", che supera ormai la rigida contrapposizione della due classi medioevali», R. BERTOLIno, «Sensus fidei», carismi e diritto nel popolo di Dio, in: L. Gerosa (a cura di), Antropologia, fede e diritto ecclesiale, Milano 1995, 90. 
Nonostante tali autorevolissime opinioni i fedeli laici, nella produzione canonistica antecedente alla codificazione del 1917, sono presentati essenzialmente dal punto di vista passivo-negativo: si definisce il laico anzitutto specificando cosa esso non è; il Wernz, già nel ricordare l'origine del termine "laico" (dal greco $\lambda \alpha o ́ \varsigma=$ popolo), ricorda che essi: «per modum stabilis terminologiae dicuntur fideles a clero distincti〉 ${ }^{12}$. Se si passa dall'etimologia ai contenuti più strettamente giuridici, vi è qualche apertura maggiore, laddove si definiscono i laici come: «omnes fideles baptizati, qui in gradu quodam in hierarchia ecclesiastica praesertim ordinis carent $\rangle^{13}$, riconoscendo dunque la comune origine battesimale di tutti i membri del "populo" christiano, ma la concessione termina quasi subito, laddove si sottolinea che: "Quare "laicus" et "subditus ecclesiasticus" opponuntur "clerico" et "Praelato ecclesiastico"»", così di fatto riprendendo la classica definizione grazianea più sopra ricordata.

Si diventa membri della Chiesa con la recezione del Battesimo, che tuttavia non comporta alcun tipo di contributo alla triplice potestà della Chiesa di governare, santificare, ed insegnare ${ }^{15}$.

Per ciò che attiene ai diritti e doveri dei laici, essi sono limitati in ambiti ben precisi e definiti:

acquirunt habilitatem recipiendi reliqua sacramenta; fiunt ministri sacramenti matrimonii, si altera pars huius sacramenti capax sit; vi baptismi recepit gaudent privilegio Paulino; pro suo iure postulant a sacerdotibus sibi constitutis certas functiones vel benedictiones liturgicas v. g. mulieres legitimo matrimonio iunctae benedictionem post partum. [...] Praeterea cum laici sensu stricto etiam extra hiererchiam iurisdictionis sint costituti, nulla ipsis competit de rebus ecclesiasticis disponendo facultas, sed tantum necessitas incumbit obediendi, nisi speciali iure aliud cautum $\operatorname{sit}^{16}$.

${ }^{12}$ F. X. WernZ, Ius decretalium, t. II, Romae 1899, 15, nota 6.

${ }^{13}$ Ibidem, 14, le sottolineature sono nel testo.

${ }^{14}$ Ibidem, 14.

${ }^{15}$ Sulla concezione odierna sul punto si veda: M. E. GonZÁLEZ, Laicos (participación en la potestad), in: J. Otaduy - A. Viana - J. Sedano (a cura di), Diccionario General de Derecho Canónico, vol. IV, Pamplona 2012, 964-968.

${ }^{16}$ F. X. Wernz, Op. cit., 17. 
In generale gli Autori che si sono occupati della condizione soggettiva dei laici nella Chiesa prima della codificazione del 1917 hanno ribadito la concezione di assoluta disuguaglianza tra chierici e laici, propria della dottrina canonistica tradizionale ${ }^{17}$.

L'impostazione del CIC17, tuttavia, se rispondeva ad esigenze di riorganizzazione e sistematizzazione del diritto canonico, allora quanto mai urgenti, data la mole e l'assoluta disorganicità raggiunta dal c.d. Corpus Iuris canonici e si poneva nel solco della tradizione canonistica più autorevole, tradiva tuttavia il concetto di persona che era stato alla base della riflessione filosofico-giuridica ed antropologica cattolica che, seppur molto raffinata sul piano teoretico, risultava assolutamente marginale. Il riferimento è al Rosmini il quale, nella celebre definizione di persona umana come «essenza del diritto» ${ }^{18}$, anticipò sicuramente $i$ tempi non ancora maturi per un salto che sarebbe avvenuto solamente un secolo dopo.

La riflessione del Vaticano II, richiedeva un ripensamento della categoria dei fedeli laici, specialmente a partire da quanto dichiarato nella Cost. Lumen gentium n. 31 e nel Decreto Apostolicam actuositatem (in partic. al n. 5) ${ }^{19}$. Non vi è qui il tempo per soffermarsi adeguatamente sui presupposti conciliari che sono alla base dei canoni oggi riguardanti i doveri e diritti dei fedeli laici, né tanto meno sui contenuti di questi specifici canoni ${ }^{20}$, ci preme invece soffermarci sul più ampio tema dei

${ }^{17}$ Sulla posizione della dottrina canonistica del XIX secolo in merito al tema dei diritti dei fedeli laici si vedano: M. BAHIMA, La condición jurídica del laico en la doctrina canónica del s. XIX, Pamplona 1972; A. LEDESMA, La condición jurídica del laico. Del C.j.c. al Vaticano II, Pamplona 1972; M. Gomez CARrasco, La condición jurídica del laico en el Concilio Vaticano II, Pamplona 1972; J. M. GonzÁlez del Valle, Derechos fundamentales y derechos públicos subjectivos en la Iglesia, Pamplona 1972; J. Hervada, Notas sobre la nocion de laico en los canonistas decimononicos, Scripta Theologica 4 (1972) 201-221.

${ }^{18}$ A. Rosmini, Filosofia del diritto, Parte I, libro I, cap. 3.

${ }^{19}$ Per una analisi di tali documenti nell'ottica della loro incidenza giuridica sui diritti dei fedeli laici, si veda: G. Lo CAstro, Il mistero del diritto. II. Persona e diritto nella Chiesa, Torino 2011, 162-174.

${ }^{20}$ Per una efficace analisi rimando al magistrale lavoro di A. Del Portillo, Laici e fedeli nella Chiesa, Milano 1999, in partic. 149 ss. 
diritti dei fedeli intesi come espressione giuridica dei valori riguardanti la persona che, in virtù di questo esplicito riconoscimento normativo, trovano adeguato spazio nell'ordinamento giuridico ecclesiale.

\section{La riforma del Codice pio-benedettino}

\subsection{Il dibattito pre-conciliare}

Gli sviluppi della teologia pre-conciliare presentano un notevole interesse anche per il tema riguardante l'approfondimento sulla figura dei laici nella Chiesa. L'analisi del dato biblico riporta l'attenzione per la persona umana al centro della riflessione teologica. Basti pensare alle affermazioni di Romano Guardini nel fondamentale capitolo $L a$ persona e Dio in Mondo e persona ${ }^{21}$, ove si afferma che

La persona ha un'importanza di senso che trascende il suo peso d'essere. In certo modo il suo senso è incondizionato. In quanto è questa persona, non in quanto è soggetto dotato di queste o quelle proprietà e doti, essa è unica, irripetibile. Come tale essa possiede una dignità e una responsabilità che nulla può surrogare ${ }^{22}$.

Affermazione che appare sicuramente stridere con quelle della scienza canonica ad essa contemporanee, e ciò che appare anche maggiormente "rivoluzionario" è il fatto che tali affermazioni siano poi suffragate dal commento dei testi paolini, dunque nel solco di una solida tradizione.

Le affermazioni della scienza canonistica dell'epoca e di parte di quella teologica ${ }^{23}$, non devono però apparire come retrograde, rispetto alle affermazioni di Guardini, ma devono essere comprese alla luce di una diversa visione delle cose, dettata dalla tradizione medievale ed

\footnotetext{
${ }^{21}$ R. Guardini, Welt und Person. Versuche zur christliche Lehre von Menschen, Mainz 1939, citiamo dalla trad. it. Mondo e persona. Saggio di antropologia cristiana, Brescia 2000.

${ }^{22}$ R. Guardini, Mondo e persona, cit., 173.

${ }^{23}$ Anche in ambito teologico non si riscontrano sostanziali differenze, il Dizionario di teologia morale del Roberti (Roma 1954) per la voce laico, rimanda alla voce chierico, ed in questa non si trova praticamente nulla che riguardi i laici.
} 
ereditata dalla scienza giuridica, che è ben evidenziata dalle parole di un altro teologo il cui pensiero fu fondamentale nel processo di riforma della Chiesa:

il mondo antico è caratterizzato dal predominio dell'aspetto oggettivo, e correlativamente da una sensibilità molto debole nei confronti del soggetto. Il mondo moderno, al contrario è caratterizzato fino in fondo dalla scoperta del punto di vista del soggetto. [...] Mentre nel mondo antico si prestava poca attenzione alla maniera in cui qualcuno faceva qualche cosa od era arrivato a qualche cosa, ma l'essenziale era la cosa stessa, il mondo moderno si interessa soprattutto alla maniera di fare le $\operatorname{cose}^{24}$.

La costituzione gerarchica della Chiesa, comincia peraltro ad essere letta e definita in maniera differente rispetto al passato proprio dal magistero; le affermazioni di Pio XII, fanno percepire una insufficienza delle impostazioni fornite sino a quel momento:

Non bisogna però credere che questa organica struttura della Chiesa sia costituita dai soli gradi della gerarchia e ad essi limitata, oppure, come ritiene un'opposta sentenza, consti unicamente di persone $c a$ rismatiche. [...] Ma giustamente i Padri della Chiesa, quando lodano i ministeri, i gradi, le professioni, gli stati, gli ordini, gli uffici di questo Corpo, hanno presenti sia coloro che furono iniziati ai sacri ordini, sia quelli che, abbracciati i consigli evangelici, menano o una vita nascosta nel silenzio o una vita che l'una e l'altra congiunge secondo il proprio istituto; sia quelli che nel secolo si dedicano con volontà fattiva alle opere di misericordia per venire in aiuto alle anime e ai corpi; infine coloro che son congiunti in casto matrimonio. [...] anche essi, ispirati e aiutati da Dio, possono ascendere al vertice della più alta santità, la quale, secondo le promesse di Gesù, non mancherà mai nella Chiesa ${ }^{25}$.

${ }^{24}$ Y. Congar, Vera e falsa riforma nella Chiesa, Milano 1994, 56 (tr. it. dell'originale francese Paris 1968). Congar fu il teologo che ebbe maggior influenza nel dibattito teologico contemporaneo sul laicato, basti pensare dapprima a Jalons pour une théologie du lä̈cat (1953, tr. it Per una teologia del laicato, Brescia 1967) e successivamente con Ministères et communion ecclésiale (1971, tr. it Ministeri e comunione ecclesiale, Brescia 1973), ove rivide in parte le sue precedenti posizioni. Accanto a Congar, possiamo ricordare almeno il contributo di K. RAHNER, Ueber das Laienapostolat, in: Schriften zur Theologie, vol. II, Einsiedeln 1955.

${ }^{25}$ PIO XII, Enciclica «Mystici corporis», n. 17, 29 giugno 1943, AAS 35 (1943) 193- 
Non è questa la sede per approfondire la vasta riflessione teologica che ha preparato l'evento conciliare ${ }^{26}$, ma preme sottolineare come la canonistica rimase in questa fase sostanzialmente refrattaria ad accogliere temi che apparivano uscire dagli schematismi disciplinati dal CIC1727. Tuttavia l'immagine della Chiesa quale Corpo mistico di Cristo, pur mantenendo fermo il principio gerarchico proprio della società ecclesiale, indicava anche l'importanza di tutte le singole membra nella edificazione dell'unico Corpo, e dunque poneva le basi per quello che, negli sviluppi del Concilio Vaticano II, diverrà il principio di uguaglianza, che si pone accanto e sullo stesso piano di quello gerarchico.

La canonistica comincia a manifestare un sostanziale mutamento di prospettive, solamente nel periodo che coincide con le ultime fasi del dibattito conciliare ed a partire dalla promulgazione della Costituzione dogmatica Lumen gentium, che per la prima volta fornisce le basi dottrinali per un superamento della concezione di Chiesa gerarchica, fino ad allora imperante ${ }^{28}$.

248. Appare quanto meno singolare notare come, nella citata Enciclica, non compaia nemmeno una volta la locuzione Popolo di Dio, che dominerà, come noto, la teologia del Vaticano II.

${ }^{26}$ Una buona sintesi dello status quaestionis si ritrova in: D. G. Astigueta, La noción de laico desde el Concilio Vaticano II al CIC '83, Roma 1999, 5-14.

${ }^{27}$ Nonostante tale contesto dottrinale, già verso la fine degli anni '40, si incomincia a parlare di diritti fondamentali dei fedeli o quanto meno di diritti subiettivi, intendendosi quei diritti che Dio ha concesso all'uomo, sulla base del diritto naturale, affinché egli possa seguire il bene ed evitare il male, conseguendo così la salvezza eterna, cf. P. Ciprotti, Personalità e battesimo nel diritto della Chiesa, Il Diritto Ecclesiastico 53 (1942) 276-281; G. Olivero, Sur le problème du droit subjectif dans le droit canonique, Ephemerides Iuris Canonici 3 (1947) 395-428; G. Olivero, Intorno al problema del diritto soggettivo nell'ordinamento canonico, Torino 1948; L. DE LuCA, I diritti fondamentali dell'uomo nell'ordinamento canonico, in: Acta Congressus Internationalis Iuris Canonici, Romae 1953, 88-103; nel medesimo Congresso vi furono altri interventi dedicati ai diritti soggettivi dei fedeli: P. CірRотті, De vocabulorum usu ad ius subiectivum designandum in Codice Iuris canonici, in: Acta Congressus Internationalis, 57-61; G. Forchielli, Precisazioni sul concetto di «persona» nel diritto canonico, in: Acta Congressus Internationalis, 127-129; W. OnCLIN, Considerationes de iurium subjectivorum in Ecclesia fondamento ac natura, in: Acta Congressus Internationalis, 211-226; G. PhILIPS, Le rôle du lä̈cat dans l'Eglise, Paris 1953.

${ }^{28}$ Cf. B. VAn LeEuwen, La partecipazione comune del Popolo di Dio all'ufficio pro- 
Vale ovviamente la precisazione del Mörsdorf riferita a certe correnti presenti nella Chiesa dell'immediato post-concilio, che hanno inteso in modo distorto le affermazioni sul principio di uguaglianza tra i fedeli applicando ad esso categorie proprie delle moderne costituzioni civili

Basandosi su affermazioni del Concilio, che vengono accuratamente selezionate secondo i propri interessi, si avanzano pretensioni che pongono in dubbio gli stessi fondamenti della costituzione ecclesiale. Rinuncio qui a mostrare i diversi errori che si divulgano in libri, riviste e congressi su temi di ecclesiologia, e che in fondo tendono a offuscare il contenuto dell'ordine sacro diminuendo così la differenza esistente tra chierici e laici. Voglio tuttavia accennare ad un fenomeno linguistico dei nostri giorni: si tratta del contrasto che si fa sorgere tra la 'Chiesa ufficiale' e la 'Chiesa dei laici', contrasto che rispecchia la concezione di certe tendenze [...] questo modo di esprimersi pone in dubbio l'unità della Chiesa [...] queste espressioni corrispondono a una concezione completamente contraria a quella del Concilio ${ }^{29}$.

\subsection{I lavori preparatori del nuovo CIC}

Come noto, sebbene la riforma del Codice di diritto canonico fosse stata annunciata in contemporanea con la volontà di indire un Concilio $^{30}$, tuttavia i lavori preparatori per la nuova codificazione cominciarono solamente dopo la promulgazione dei documenti conciliari ${ }^{31}$,

fetico di Cristo, in: G. Baraúna (a cura di), La Chiesa del Vaticano II. Studi e commenti intorno alla Costituzione dommatica «Lumen gentium», Firenze 1965, 481-490; E. HAMEL, Aequalitas fundamentalis omnium christifidelium in Ecclesia secundum Concilium Vaticanum II, Periodica 63 (1967) 247 ss.; M. Petroncelli, Il diritto canonico dopo il Concilio Vaticano II, Napoli 1969; P. Gismondi, Il diritto della Chiesa dopo il Concilio, Milano 1973.

${ }^{29}$ K. MörSDORF, Das eine Volk Gottes und die Teilhabe der Laien an der Sendung der Kirche, in: K. Siepen - J. Weitzel - P. Wirth (a cura di), Ecclesia et Ius. Festgabe für A. Scheuermann zum 60, Munchen-Paderborn-Wien 1968, 99, la traduzione italiana si trova in: A. CATTANEO, Questioni fondamentali della canonistica nel pensiero di Klaus Mörsdorf, Pamplona 1986, 231.

${ }^{30}$ GiovannI XXIII, Primus Concilii Oecumenici nuntius, 25 gennaio 1959, AAS 51 (1959) 65-69.

${ }^{31}$ La Pontificia Commissio Codici Iuris Canonici recognoscendo, venne costituita 
e ciò per una precisa scelta della Pontificia Commissione designata, che volle attendere gli sviluppi conciliari prima di intraprendere un lavoro che avrebbe altrimenti corso il rischio di risultare poi superato.

La visione data dal Concilio della Chiesa come popolo di $\mathrm{Dio}^{32}$, e le conseguenti affermazioni riguardanti il principio gerarchico posto in stretta correlazione con il principio di uguaglianza fondamentale di tutti i fedeli, comportò l'impellente necessità di modificare in modo sostanziale il CIC17 proprio nella parte riguardante le persone; in particolare risultava assolutamente non più adeguata la precedente suddivisione per status del popolo di Dio in quanto contraddiceva il citato principio di uguaglianza fondamentale derivante dal sacramento battesimale. Inoltre il Concilio mutò la prospettiva ecclesiologica, con il passaggio da una ecclesiologia gerarcologica ad una ecclesiologia di tipo comunionale, il che non poteva non avere dirette conseguenze circa il modo di intendere il fedele, ed il laico in particolare, in ambito giuridico-canonico ${ }^{33}$.

il 28 marzo 1963 e tenne la sua prima riunione il 12 novembre dello stesso anno, cf. Communicationes 1 (1969) 35. Per una breve storia dei lavori preparatori del CIC83, si vedano, fra gli altri: F. D'Ostilio, La storia del nuovo Codice di Diritto Canonico. Revisione-Promulgazione-Presentazione, Città del Vaticano 1983; J. L. GutiÉrRez, La formazione dei principi per la riforma del "Codex Iuris Canonici", in: J. Canosa (a cura di), I Principi per la revisione del Codice di Diritto Canonico. La ricezione giuridica del Concilio Vaticano II, Milano 2000, 5-29.

${ }^{32}$ Tale concetto ecclesiologico è per altro presente nella dottrina canonistica anche anteriore al Concilio Vaticano II, in particolare vedi: E. Eichmann - K. Mörsdorf, Lehrbuch des Kirchenrechts, Paderborn $1947^{5}$. In tale manuale si spiegva tra l'altro che: «La Chiesa è il nuovo Popolo di Dio, che vive in un ordine gerarchico, per realizzare il Regno di Dio sulla terra», 21; inoltre nel pensiero di Mörsdorf, la Chiesa trovava la sua denominazione ideale proprio nel concetto di popolo di Dio: «tra le diverse denominazioni della Chiesa, le uniche che abbiano un significato oggettivo, sono quelle

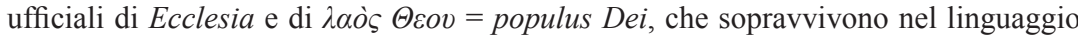
liturgico», Op. cit., XI ed., 9.

${ }^{33}$ R. J. Castillo Lara, La communion ecclesiale dans le nouveau Code de Droit Canonique, Studia Canonica 17 (1983) 331-335; J. Herranz, Génesis del nuevo cuerpo legislativo de la Iglesia, Ius Canonicum 23 (1983) 491-526. Per gli aspetti teologici, si veda: J. L. Llanes, La discusión teológica sobre la noción de laico, Scripta Theologica 22 (1990) 777-782. 
Fin dal 1967, con le prime riunioni del Coetus centralis della Commissione per la revisione del CIC17, appare chiaro che il tema dei diritti e doveri dei fedeli (laici compresi) è tra le tematiche di maggior rilievo ${ }^{34}$. Come noto i Principia furono presentati all'Assemblea generale del Sinodo dei Vescovi del 1967 e da essa approvati ${ }^{35}$; fra di essi il sesto principio riguardante De tutela iurium personarum, esplicitamente prevedeva, tra i criteri guida dei lavori di codificazione:

[...] Unicuique christifidelium iura agnoscenda ac tuenda sunt, et quae in lege naturali vel divina positive continentur, et quae ex illis congruenter derivantur ob insitam socialem conditionem quam in Ecclesia acquirunt et possident.

[...] ut in futuro Codice ob radicalem aequalitatem quae inter omnes chrstifideles vigere debet, tum ob humanam dignitatem tum ob receptum baptisma, statutum iuridicum omnibus commune condatur, antequam iura et official recenseantur quae ad diversas ecclesiasticas functiones pertinent $t^{36}$.

Una ulteriore indicazione circa il riconoscimento dei diritti dei fedeli, veniva pure dal settimo principio (De ordinanda procedura ad tuenda iura subiectiva), il quale sottolineava l'importanza di strutturare il nuovo sistema processuale dando la possibilità a tutti i fedeli di veder tutelati i propri diritti in sede giudiziale ${ }^{37}$.

${ }^{34}$ Conosciamo in particolare il contenuto del voto espresso nell'anno 1966 sul tema da uno dei consultori del Coetus, Alvaro Del Portillo, in quanto fu pubblicato nella prima edizione spagnola del volume: Fieles y laicos en la Iglesia. Bases de sus respectivos estatutos jurídicos, Pamplona 1969, 152.

${ }^{35} \mathrm{Il}$ testo dei Principia quae Codicis Iuris Canonici recognitionem dirigant si trova in: Communicationes 1 (1969) 77-100; il testo latino con traduzione italiana a fronte si trova in: Enchiridion Vaticanum 2, 1358-1377.

${ }^{36}$ Communicationes 1 (1969) 82-83.

${ }^{37}$ È noto che tale principio rimase in parte inapplicato, proprio per ciò che riguardava la possibilità di ottenere la tutela dei propri diritti, in sede amministrativa, da parte di tutti i fedeli. L'analisi delle motivazioni ci porterebbe lontano dal nostro tema, si vedano sul punto, tra gli altri: R. Bertolino, La tutela dei diritti nella Chiesa. Dal vecchio al nuovo Codice di Diritto Canonico, Torino 1983; J. Llobell, Il sistema giudiziario canonico di tutela dei diritti. Riflessioni sull 'attuazione dei Principi $6^{\circ}$ e $7^{\circ}$ approvati dal Sinodo del 1967, in: I principi per la revisione del Codice di Diritto Canonico, cit., 501-546. 
Iniziati così a pieno ritmo i lavori codificatori, seguirono anche numerose pubblicazioni di approfondimento sui diritti e doveri dei fedeli in generale e sui diritti dei fedeli laici ${ }^{38}$. È proprio con riferimento a tale tematica, che si possono apprezzare le differenti visioni proprie delle diverse tendenze che più hanno influenzato la scienza canonistica contemporanea ${ }^{39}$.

Abbondantissima risulta la produzione sul tema da parte dei canonisti dell'Università di Navarra, anzitutto ad opera dei due "padri fondatori": Pedro Lombardia e Javier Hervada. Testo sicuramente fondamentale rimane, su tale tematica, El Derecho del Pueblo de Dios. Hacia un sistema de derecho canonico (Pamplona 1970), in due volumi, scritto a quattro mani dai due citati Autori; in esso si tratta il tema dei diritti e doveri fondamentali dei fedeli, sulla base del principio di uguaglianza fondamentale tra tutti i membri del popolo di Dio, chierici e laici, alla luce delle affermazioni del Concilio Vaticano $\mathrm{II}^{40}$. Numerosi furono anche gli studi dedicati al tema dei diritti dei fedeli dai canonisti della scuola di Monaco ${ }^{41}$. Tra i canonisti della Scuola Laica Italiana, si se-

${ }^{38} \mathrm{Si}$ trova un'abbondante bibliografia nel citato: D. G. AstiguetA, La noción de laico desde el Concilio Vaticano II al CIC '83, cit.

${ }^{39}$ Preferiamo parlare di "tendenze", piuttosto che di "scuole", in quanto i confini tra i diversi orientamenti appaiono oggi assai meno definiti e rigidi di quanto non apparisse in passato, cf.: A. CATtaneo, Fondamenti ecclesiologici del diritto canonico, con la collaborazione di C.-M. Fabris, Venezia 2011, 60-63.

${ }^{40}$ Non si può peraltro dimenticare che tale testo, arriva tuttavia dopo due precedenti lavori sul tema, sempre ad opera di due canonisti della medesima Università: Alvaro Del Portillo con il già citato Fieles y laicos en la Iglesia. Bases de sus respectivos estatutos jurídicos, (Pamplona 1969, tr. it. Laici e fedeli nella Chiesa, Milano 1999²) e e Pedro-Juan Viladrich, la cui prima produzione scientifica fu dedicata alle questioni fondamentali della canonistica con il volume Teoría de los derechos fundamentales del fiel. Presupuestos críticos (Pamplona 1969, con introduzione di P. Lombardia).

${ }^{41}$ Oltre ai testi già citati alle note precedenti, si vedano: K. Mörsdorf, Grundfragen einer Reform des kanonischen Rechtes, Münchener Theologische Zeitschrift 15 (1964) 1-16; K. Mörsdorf, Die Zusammenarbeit von Priestern und Laien in ekklesiologischkanonistischer Sicht, Veröffentlichungen der Katholischen Akademie der Erzdiözese Freiburg 11 (1968) 13-26; K. MöRSDORF, Das konziliare Verständnis vom Wesen der Kirche in der nachkonziliaren Gestaltung der kirchlichen Rechtsordnung, Archiv für katholischen Kirchenrecht 144 (1975) 387-401. Per una sintesi del pensiero del mae- 
gnala la posizione di Pio Fedele, il quale rimase nettamente contrario alla possibilità di riconoscere l'esistenza di diritti soggettivi all'interno dell'ordinamento giuridico ecclesiale, in quanto tale ordinamento, essendo di natura sostanzialmente divina, non avrebbe potuto ospitare al suo interno elementi propri di ordinamenti giuridici statuali, quali il diritto privato, l'autonomia, la libertà e gli interessi privati dei singoli fedeli ${ }^{42}$.

Vi era poi la posizione di chi si dimostrava contrario all'introduzione della categoria dei diritti e doveri fondamentali dei fedeli, a motivo del fatto che una prospettiva di diritto naturale nel diritto della Chiesa, comporterebbe una falsificazione del dato ecclesiologico, dando così alla nozione di diritto naturale una visione di chiaro stampo illuministico ${ }^{43}$.

Durante i primi anni dei lavori codificatori l'attenzione del Coetus che si occupava dei laici si concentrò sulla preparazione di una definizione di laico, che potesse trovare spazio all'interno di un'apposita sezione del nuovo Codice ${ }^{44}$, sottolineando in particolare l'importanza dell'"indole secolare", ed accogliendo le tesi proposte dai consultori Del Portillo e Lombardia, i quali volevano che il Codice riproponesse le tesi conciliari (in particolare quelle di LG n. 31 e AA) ${ }^{45}$.

stro monacense sul tema si veda: A. CATTANEO, Questioni fondamentali della canonistica nel pensiero di Klaus Mörsdorf, 227-288. Tra gli allievi di Mörsdorf si ricordano: E. Corecco, La «sacra potestas» e i laici, Studi Parmensi 28 (1980), 3-36, ora in: G. Borgonovo - A. Cattaneo (a cura di) Ius et Communio. Scritti di Diritto Canonico, vol. II, Casale Monferrato 1997, 249-282; W. Aymans, «Munus» und "sacra potestas», in: E. Corecco - N. Herzog - A. Scola (a cura di), I diritti fondamentali del cristiano nella Chiesa e nella società, Friburgo-Milano 1981, 185-202; A. M. Rouco VArela, Fundamentos eclesiológicos de una teoría general de los derechos fundamentales del cristiano en la Iglesia, in: I Diritti Fondamentali del Cristiano, cit., 53-78.

${ }^{42}$ Tale prospettiva appartiene già al celebre P. FedeLE, Discorso generale sull'ordinamento canonico, Padova 1941, ma si riscontra sostanzialmente invariato in tutta la successiva produzione dell'Autore, che si rifaceva all'autorevole posizione di Jemolo. In tale prospettiva si inserisce pure P. Bellini, Diritti fondamentali dell'uomo diritti fondamentali del cristiano, Ephemerides Iuris Canonici 34 (1978) 211-246.

${ }^{43}$ P. Hinder, Grundrechte in der Kirche. Eine Untersuchung zur Bergründung der Grundrechte in der Kirche, Freiburg S. 1977.

${ }^{44}$ Sugli sviluppi dei lavori preparatori si veda: L. B. RiBé, El concepto de laico en el coetus "De laicis" en la codificación de 1983, Roma 1994.

${ }^{45}$ Le varie definizioni di fedele laico elaborate nei lavori preparatori si trovano in: 
Successivamente, a partire dalla VI sessione dei lavori del Coetus, si ebbe un mutamento di prospettiva, che portò alla decisione di eliminare la definizione di laico, per concentrarsi solamente sui doveri e diritti dei fedeli laici, intesi quali non chierici e non religiosi ${ }^{46}$; sebbene non vi siano spiegazioni a tale mutamento di prospettive, si può forse ipotizzare che le vicende riguardanti l'elaborazione della Lex Ecclesiae Fundamentalis $[=L E F]$ si siano in parte sovrapposte con quelle relative alla codificazione dei doveri e diritti dei fedeli laici, sebbene i progetti di $L E F$ non contenessero specifiche norme di tipo definitorio riferibili ai laici.

\section{Il dibattito sui diritti fondamentali}

Il Congresso internazionale di diritto canonico che si svolse nel 1980 a Friburgo (Svizzera), all'indomani della promulgazione del nuovo Codice di diritto canonico, fu dedicato al tema dei diritti fondamentali dei fedeli ${ }^{47}$. Fra le diverse questioni dibattute ci fu anche quella dell'opportunità o meno di usare l'espressione «diritti fondamentali» all'interno del nuovo Codice di diritto canonico; in particolare al centro del dibattito fu l'utilizzo dell'aggettivo «fondamentali» applicato ai diritti dei fedeli.

Tutto il dibattito iniziò a svilupparsi sulla scia dell'elaborazione della $L E F^{48}$, contestualmente all'inizio dei lavori di codificazione canonica. Tale $L E F$ avrebbe dovuto essere, nelle intenzioni del legislatore, un testo di natura costituzionale, sul modello di quelli in uso presso i moderni Stati civili, contenente tutte le norme riguardanti i diritti ed

Communicationes 17 (1985) 173-174 e Communicationes 18 (1986) 351.

${ }^{46} \mathrm{Cf}$. Communicationes 18 (1986) 376.

${ }^{47}$ Gli atti del Convegno sono ora raccolti nel volume: E. CoreCCO - N. HerzoG - A. Scola (a cura di), I diritti fondamentali del cristiano nella Chiesa e nella società, Friburgo - Milano 1981.

${ }^{48}$ Sulla storia e sui contenuti di tale progetto normativo si veda il fondamentale lavoro di D. Cenalmor, La Ley Fundamental de la Iglesia. Historia y análisis de un proyecto legislativo, Pamplona 1991, e l'amplia bibliografia ivi contenuta, ed ora anche: D. CENALMOR, Ley Fundamental de la Iglesia, in: Diccionario General de Derecho Canónico, cit., vol. V, 80-87. Per i testi dei due primi schemi della LEF (maggio 1969 e luglio 1970) si veda: O. G. M. Boelens, Synopsis “Lex Ecclesiae Fundamentalis”, Lovanio 2001. 
i doveri dei fedeli e la costituzione gerarchica della Chiesa, ed altre ancora. Tale testo sarebbe stato comune per tutta la Chiesa, sia latina che orientale, ed avrebbe dovuto essere di rango superiore ad ogni altra norma ecclesiale, compreso lo stesso Codice canonico, su esplicita indicazione del Santo Padre ${ }^{49}$.

Tuttavia, in corso d'opera, il progetto divenne di pubblico dominio e suscitò le accese critiche di una parte del mondo canonistico e non solo $^{50}$. Le critiche, non prive di una forte impronta ideologica, tipica di quegli anni, vertevano anzitutto sui contenuti del progetto di legge che era ritenuto un passo indietro rispetto alle prospettive aperte dal dibattito conciliare ${ }^{51}$. Il progetto suscitò, proprio in ambito canonistico, un'ampia discussione dottrinale e vide sostanzialmente contrapposti due schieramenti di favorevoli (non senza opportune precisazioni) e contrari a tale progetto ${ }^{52}$.

${ }^{49}$ «Peculiaris vero hic exsistit quaestio eaque gravis, eo quod duplex est Codex Iuris Canonici, pro Ecclesia Latina et Orientali, vide licet num conveniat comune et fundamentalem condi Codicem, ius constitutivum Ecclesiae continentem», PAOLO VI, Discorso ai membri della Pontificia Commissione per la Revisione del Codice di Diritto Canonico, AAS 57 (1965) 988.

${ }^{50}$ Il 10 aprile 1970 la rivista National Catholic Reporter di Kansas City, pubblicò il Textus Prior (del 1969) dello schema di LEF, dichiarando che si trattava di un documento segreto e con una forte connotazione anti ecumenica; la pubblicazione veniva accompagnata da un simposio, di tre giorni, dal titolo: Corresponsability in the Church, tenutosi presso il Cathedral College a Duglaston (Long Island - NY), con il patrocinio della Canon Law Society of America e l'aiuto della Fordham University. Particolarmente critico fu H. Küng, il quale dichiarò che il progetto creava una «confusione tra il dogma e la legge, una dogmatizzazione della legge e una legalizzazione del dogma»; da conto delle critiche al progetto il volume Redaccion Ius Canonicum (a cura di), El Proyecto de Ley Fundamental de la Iglesia texto bilingüe y análisis crítico, Pamplona 1971, che presenta il Textus emendatus (1970) del progetto di LEF, e diversi interventi di commento, in particolare sulla divulgazione del progetto si veda: J. M. Gonzalez del Valle, La critica al proyecto, cit., 69-74, da cui la citazione di H. Küng (p. 69). Il testo del progetto di $L E F$, venne pubblicato pure in: Il Regno (10 luglio 1970) 284-300 e Herder-Korrespondenz 24 (1970) 272-281.

${ }^{51}$ AA. Vv., Legge e Vangelo. Discussione su una legge fondamentale per la Chiesa, Brescia 1972, in cui si segnala, in particolare, l'intervento critico di G. Alberigo, Fede, istituzione e Lex fundamentalis nella tradizione cristiana, 15 ss.

${ }^{52} \mathrm{Si}$ vedano: J. Beyer, De Legis Ecclesiae Fundamentalis. Redactione, natura et 
Semplificando qui notevolmente $\mathrm{i}$ termini della questione basterà ricordare che i favorevoli, oltre a sottolineare la necessità di un testo comune per la Chiesa latina e per le Chiese orientali, sostenevano l'importanza di un corpo di norme con carattere costituzionale, aventi origine nel diritto divino. I contrari sottolineavano il rischio di fossilizzare un tale tipo di norme, per loro natura dinamiche, in un testo legislativo, avvertendo il pericolo di una confusione tra il dato strettamente giuridico ed il dato teologico.

Per quanto attiene più specificatamente il tema che qui ci occupa, la $L E F$ conteneva un elenco dei diritti e doveri fondamentali di tutti i fedeli ed il dibattito si concentrò su questo particolare aspetto del progetto. Da un lato si sosteneva la correttezza dell'impostazione metodologica adottata dalla $L E F$, che riconosceva valenza costituzionale ai diritti dei fedeli, sottolineando il valore fondamentale di tali diritti, quasi sul modello di quanto avveniva nelle costituzioni dei moderni Stati nazionali. I critici sostenevano, al contrario, che tale prospettazione era assolutamente errata, in quanto non era possibile applicare alla Chiesa ed al suo diritto categorie proprie del diritto civile; inoltre, i di-

crisi, Periodica 61 (1972) 525-551; W. Bertrams, Communio, communitas, societas in Lege Fundamentali Ecclesiae, Periodica 61 (1972) 553-604; L. Vela-SÁncheZ, Christifidelium officia et iura fondamentalia descripta in Legis Fundamentalis Schematis textu emendato, Periodica 61 (1972) 605-623; C. CoRRAL, De relatione inter Ecclesiam et Statum in Schemate Legis Ecclesiae Fundamentalis, Periodica 61 (1972) 625-645; J. Manzanares, De Schemate Legis Ecclesiae Fundamentalis in Colloquio HispanoGermanico adnotationes, Periodica 61 (1972) 647-662; M. M. WoJnAR, Relatio de crisi Legis Fundamentalis in Statibus Foederatis Americae Septentrionalis, Periodica 61 (1972) 663-670; A. Moroni (a cura di), Lex fundamentalis Ecclesiae, Atti della tavola rotonda, Macerata 12-13 ottobre 1971, Milano 1973, dalle cui relazioni appaiono favorevoli, pur non senza riserve, al progetto di $L E F$ : O. Giacchi, Gismondi, P. Lombardia, A. Ravà, G. Leclerc; AA. Vv., Lex Ecclesiae Fundamentalis, Roma 1974, con interventi di P. Fedele, J. Beyer e P. Lombardia, dei quali il primo sostanzialmente contrario al progetto, ma per ragioni assai differenti rispetto a quelle esposte dall'Alberigo (ciritcato da P. Fedele). Si vedano pure: K. Mörsdorf, Lex Ecclesiae Fundamentalis. Bericht über die Arbeitsergebnisse eines Kanonistischen Symposions 1971, Archiv für katholischen Kirchenrecht 140 (1971) 410-496; K. Mörsdorf, De Legis Ecclesiae Fundamentalis condendae sensu et fine, in: Conventus Canonistarum hispano-germanus, Salamanca 1974, 47-61. 
ritti e doveri fondamentali dei fedeli, avrebbero rispecchiato in modo acritico i diritti e doveri dei cittadini, che tuttavia avevano un'origine illuministica che si poneva in netto contrasto con i contenuti teologici sottostanti alla costituzione della Chiesa.

Il dibattito intorno ai diritti e doveri fondamentali dei fedeli, così come espressi nella $L E F$, divenne un vero e proprio scontro tra le due opposte tendenze della canonistica, e toccò il suo momento culminante in occasione del citato Congresso di Friburgo $(\mathrm{CH})$ nell'ottobre del 1980, allorquando aveva visto la luce, in pochi lo ricordano, lo Schema Postremum (gennaio 1980) della LEF. Si era, insomma, alle soglie del termine dei lavori di codificazione del nuovo CIC, ed era inevitabile un intenso dibattito sull'opportunità o meno di promulgazione di una Legge Fondamentale per la Chiesa, che costituzionalizzasse diritti e doveri dei fedeli. La sintesi delle due differenti posizioni fu espressa da un lato da P. Lombardia ${ }^{53}$, e dall'altro da E. Corecco ${ }^{54}$.

Lombardia, ribadì una volta di più la necessità del riconoscimento di diritti e doveri dei fedeli nell'ambito dell'ordinamento giuridico ecclesiale, insistendo sulla necessità di qualificare tali diritti e doveri come fondamentali, in quanto appartenenti ad un ambito costituzionale, sul modello delle dichiarazioni di principio proprie delle moderne costituzioni; non senza sottolineare le peculiarità proprie derivanti dal fatto che, sebbene di natura costituzionale, tali diritti sarebbero pur sempre inseriti nell'ordinamento di una società del tutto peculiare quale è la

${ }^{53}$ P. Lombardia, Los derechos fundamentales del cristiano an la Iglesia y en la sociedad, in: I Diritti Fondamentali del Cristiano, cit., 15-31. Sulla posizione di P. Lombardia rispetto alla nuova codificazione canonica, si vedano: J. Herranz, Il Prof. Pedro Lombardía e la nuova codificazione canonica, Ius Canonicum 52 (1986) $507-$ 513; V. Gomez-Iglesias C., El Prof. Pedro Lombardia y el proyecto de «Lex Ecclesiae Fundamentalis», Fidelium Iura 7 (1997) 103-178.

${ }^{54}$ E. Corecco, Considerazioni sul problema dei diritti fondamentali del cristiano nella Chiesa e nella società. Aspetti metodologici della questione, in: I Diritti Fondamentali del Cristiano, cit., 1207-1234. Sull'importanza delle personalità di Corecco e Lombardia per la scienza canonistica si veda: A. DE LA HerA, Dos maestros de la ciencia canónica de la segunda mitad del siglo XX. Perfil humano y personalidad científica de Pedro Lombardía y Eugenio Corecco, in: J. I. Arrieta - G. P. Milano (a cura di), Metodo, fonti e soggetti del diritto canonico, Città del Vaticano 1999, 19-31. 
Chiesa. In quest'ottica per Lombardia diventava centrale la formulazione di una Legge, con connotati di tipo costituzionale, comune tanto alla legislazione latina che a quella orientale, nel cui ambito dovevano trovare la loro formulazione anche i diritti e doveri fondamentali propri dei membri del popolo di Dio.

Corecco partiva invece da una considerazione affatto differente: il diritto della Chiesa poggia su basi soprannaturali, ed in tale ottica è impossibile il riconoscimento di diritti dell'uomo. Tale riconoscimento implicherebbe, infatti, l'ingresso nel diritto della Chiesa di categorie proprie del diritto statuale, quali quello di "società giuridicamente organizzata" e di "legge naturale", intesi alla stregua di quanto avviene nell'ambito degli ordinamenti giuridici civili. Per Corecco non era dunque concepibile l'utilizzo della categoria di diritti fondamentali, potendosi al più parlare di doveri e diritti del fedele senza ulteriori specificazioni; Corecco fece inoltre notare come l'espressione "fondamentali", riservata ad alcune norme, ed in particolare ai diritti dei fedeli, non fosse adeguata alla realtà della Chiesa. Essa porterebbe, infatti, a considerare tali diritti alla stregua di come sono considerati nella società civile: la persona umana ha una dignità e dei diritti fondamentali che sono anteriori a quelli dello Stato e che perciò ogni Stato deve riconoscere. In caso contrario, il cittadino ha il diritto di reclamarli e di denunciare l'autorità dello Stato che non li rispetta. Inoltre, nella Chiesa il rapporto fedele-Chiesa è essenzialmente diverso da quello persona-Stato: il fedele non è anteriore alla Chiesa e non ha dei diritti da far valere di fronte alla Chiesa. In caso contrario si rischierebbe di promuovere una mentalità rivendicazionistica ben poco ecclesiale $\mathrm{e}$ che non esprimerebbe pienamente l'indole comunionale propria della Chiesa; in questo senso egli negò decisamente anche la possibilità, caldeggiata fortemente da Lombardia, di strumenti giuridici diretti alla tutela dei diritti dei fedeli, quali, ad esempio, i ricorsi di tipo amministrativo.

Un altro grosso problema riguardava la posizione di norme sovraordinate che sarebbe stata riservata alla $L E F$ : data la difficoltà di definire le norme di diritto divino, la immodificabilità delle norme fondamen- 
tali, in quanto gerarchicamente superiori, sarebbe stata inaccettabile, precludendo la possibilità di una definizione maggiormente elastica. Il progetto, come noto fu accantonato, senza che ufficialmente si siano mai rese note le motivazioni di tale accantonamento, le quali restano ad oggi solamente intuibili.

\section{Il valore persona umana alla base di obblighi e diritti dei fedeli}

Oltre all'abbandono di un progetto di una norma di natura costituzionale nella Chiesa, pure l'espressione «diritti fondamentali» non è stata usata né dal CIC, né da testi magisteriali. È bene però aggiungere un dato di assoluta importanza circa il senso più profondo rappresentato dai doveri e dai diritti dei fedeli nell'ordinamento canonico.

L'ordinamento giuridico della Chiesa ha alla base dei valori, piuttosto che degli interessi, per quanto legittimi essi possano essere. Il fedele che si accosta al diritto ecclesiale e al "catalogo" dei doveri e dei diritti in esso contenuti, dovrà soprattutto guardare ai valori che stanno alla base di detto ordinamento, al significato in virtù del quale tali diritti e doveri sono stabiliti. Possiamo affermare che diritti e doveri dei fedeli hanno un carattere teleologico ${ }^{55}$, nel senso che essi devono sempre essere considerati in rapporto con i valori che ne sono alla base. Intendere diversamente tali diritti e doveri, distorcerebbe completamente il loro senso all'interno dell'ordinamento canonico, per trasformarli (specialmente i diritti) in vuote rivendicazioni dei fedeli nei confronti dell'autorità legislativa, prospettiva quest'ultima estranea al diritto ecclesiale. In questo senso le osservazioni mosse da Corecco appaiono sicuramente corrette, sebbene egli radicalizzò la sua posizione anche oltre tale limite.

Alla base dei diritti dei fedeli, siano essi chierici, religiosi o laici, vi è un valore assolutamente unico e fondamentale: 1'uomo; solo se si pone l'uomo alla base di un insieme di diritti e di doveri, tali diritti e doveri saranno in grado di esplicare al meglio la loro finalità ultima. I diritti, così intesi, saranno veramente espressione di giustizia in quanto posti a garanzia dell'uomo, e per converso i doveri saranno realmen-

${ }^{55}$ Dal greco $\tau \varepsilon \dot{\lambda} \mathrm{s}=$ fine e $\lambda$ ó $\gamma$ o $\varsigma$ discorso: scienza dei fini. 
te equi in quanto volti a porre dei limiti in vista di una migliore salvaguardia dell'uomo. Diritti e doveri diventano le "vie" fondamentali per condurre l'uomo verso il suo fine ultimo: la salvezza eterna; nonché per dar vita ad una società (la Chiesa) che sia più giusta ed equa.

Se si valutassero le norme giuridico-canoniche aventi ad oggetto diritti e doveri dei fedeli, solamente per il loro contenuto normativo-positivo, perdendo di vista il valore che tutelano, esse non potrebbero mai essere correttamente intese ed applicate; diverrebbero, come più sopra ricordato, semplici momenti di rivendicazione vuoi dei fedeli (per quanto attiene ai diritti) vuoi dell'ordinamento ecclesiale (per quanto attiene agli obblighi).

Se invece diritti e doveri dei fedeli, vengono rettamente intesi come espressione del valore uomo, che l'ordinamento giuridico ecclesiale intende compiutamente tutelare, ecco che essi saranno in grado di svolgere pienamente la loro peculiare funzione.

Gli obblighi e diritti dei fedeli elencati nel Codice, costituiscono una valutazione compiuta dal legislatore canonico circa la gerarchia da attribuire a determinati valori che l'ordinamento giuridico della Chiesa intende tutelare, ma affinché tale gerarchia di valori non sia puro arbitrio dell'autorità, essi dovranno pur sempre essere espressione del valore primario da essi tutelato che, come detto, è l'uomo inteso come imago Dei, e non in senso meramente naturalistico. In ciò la visione antropologica propria dell'ordinamento giuridico ecclesiale, si differenzia notevolmente dalla visione propria degli ordinamenti giuridici statuali, che pure possono a volte avvicinarsi ad una visione simile. Tale prospettiva appare peraltro, come visto, la prospettiva classica del diritto ecclesiale e della teologia cattolica, i quali hanno sempre inteso tutelare e valorizzare l'uomo in virtù della sua generazione divina.

Gli obblighi e i diritti di tutti i fedeli ${ }^{56}$ rappresentano uno dei punti in cui l'ordinamento canonico esprime al massimo grado il rapporto

\footnotetext{
${ }^{56} \mathrm{Nel}$ rispondere alla domanda sul perché nel CIC si parli prima dei doveri e poi dei diritti dei fedeli il Card. Castillo Lara rispondeva: «Con questa scelta il legislatore ha forse voluto, mi sembra, sottolineare che, chiamato da Dio a formar parte del suo popolo, il cristiano ha il fondamentale dovere di corrispondere alla chiamata, non solo accettando di entrare nella Chiesa, ma corrispondendo a tale chiamata durante tutta la sua esistenza, partecipando cioè attivamente alla vita ed alla missione della Chiesa, ciò
} 
tra le norme positive e quelle di diritto divino, dal momento che tali diritti e obblighi sono volti a regolamentare tutte quelle caratteristiche proprie del fedele il quale rappresenta il punto focale di tutto l'ordinamento, e dunque ne costituiscono un vero e proprio statuto giuridico.

L'importanza di tali norme, inserite in seguito alla mancata approvazione del progetto della $L E F$ nel Codice, è evidente ed è stato sottolineato da più parti, anche da chi si era espresso in modo sfavorevole alla citata $L E F^{57}$. Da più parti si è evidenziata la grande rilevanza dei canoni 208-223 i quali, pur non distinguendosi dall'intero corpo normativo e non avendo un rilievo costituzionale al pari di quanto sarebbe accaduto se fosse stata promulgata la $L E F$, hanno tuttavia un indiscutibile valore primario in quanto molti dei doveri e dei diritti espressi da tali canoni si fondano sul diritto divino ${ }^{58}$. Hervada ha sottolineato, da parte sua, l'importanza delle norme inserite in questa prima parte del Libro II del CIC e la necessità della loro prevalenza su qualsiasi altra, anche dal punto di vista pratico, tanto che sottolinea come, pur avendo lo stesso rango normativo delle altre, tuttavia

in quanto sono di diritto divino, hanno una forza tale, che la legislazione positiva deve interpretarsi conformemente a questi [canoni n.d.r.], affinché prevalgano sulle norme umane che non siano coerenti con questi, e i giudici devono risolvere i casi in modo che i diritti si riconoscano e si garantiscano ${ }^{59}$.

che rappresenta un dovere prima che un diritto», R. CASTILlo LARA, I doveri e $i$ diritti dei christifideles, Salesianum 48 (1986) 319.

${ }^{57}$ Per una analisi della particolare posizione assunta da Corecco, contrario alla definizione di fondamentali, con riguardo ai diritti dei fedeli, si veda: D. CENALMor, Los derechos fundamentales en el ámbito canónico. Origen y términos de la discusión, Fidelium Iura 15 (2005) 11-32.

${ }^{58}$ Cf. P. Lombardía, Lecciones de Derecho Canónico, Madrid 1984, 81-82. Si veda anche nel medesimo senso: J. FornÉs, El principio de igualdad en el ordenamiento canónico, Fidelium Iura 2 (1992) 118 ss., in particolare l'A. sottolinea l'importanza di tali canoni in quanto in essi «si tratta di diritti innati - iura nativa - alla condizione di libertà e di dignità del fedele [...] anteriori a qualsiasi formalizzazione giuridicopositiva; e, pertanto, con la caratteristica di essere diritti costituzionali, come dire, che integrano la costituzione stessa della Chiesa», 137 [la traduzione italiana è nostra n.d.r.].

${ }^{59}$ J. Hervada, Commentario sub Tit. I, P. I, Lib. II del CIC, in: Código de Derecho 
Aggiungiamo una ulteriore specificazione: tali diritti ed obblighi, non possiedono una particolare valenza solamente in quanto, nella gerarchia delle norme positive canoniche, possiedono una valenza di tipo costituzionale e fondamentale, ma in quanto sono conseguenza di un valore che già di per sé si pone come un valore fondamentale e primario, a prescindere dal fatto che vi siano norme positive che lo tutelano. Tali norme tutelano dei valori che sono connaturati alla persona umana e che non possono che esserle riconosciuti anche da un punto di vista giuridico. È Dio stesso che, avendo creato l'uomo a sua immagine, gli ha conferito una particolare natura che fa si che ad esso si debbano attribuire determinati diritti ed obblighi.

\section{Alcuni fondamentali canoni alla base dei diritti di tutti i fedeli}

$\mathrm{Ci}$ soffermiamo dunque principalmente su alcuni dei canoni relativi ai doveri e diritti di tutti i fedeli, in quanto costituiscono una sorta di presupposto necessario alla successiva elencazione fatta nel Libro II. Tali canoni rappresentano premesse necessarie anche per comprendere il senso dei successivi diritti ed obblighi, dal momento che affrontano questioni che si pongono alla base dei medesimi.

Il can. $207 \S 1$ specifica in che senso debba essere intesa la costituzione gerarchica della Chiesa. Il canone in esame non elenca i requisiti necessari a tale differenziazione, né stabilisce una differenziazione di valore tra $\mathrm{i}$ fedeli, basata sulla loro condizione personale. In esso si sottolinea invece l'esistenza, «per istituzione divina», di diverse «modalità» di essere dei fedeli, modalità che dipendono da scelte indipendenti e personali, e che caratterizzano l'essere stesso del soggetto che tali scelte compie. Questa libera scelta del fedele, questa modalità di essere, non comporta una sua maggiore o minore importanza nell'ambito della società ecclesiale, ma solamente un diverso ruolo all'interno di essa.

Nel momento attuale si è portati a pensare alla diversità come ad un elemento discriminatorio, laddove si pretenderebbe di trasformare la società in un unico blocco monolitico, nel quale ciascuno dovreb-

Canónico. Edicion anotada, Pamplona 1983 [la traduzione italiana è nostra n.d.r.]. 
be essere assolutamente uguale all'altro, con i medesimi diritti (meno spesso con i medesimi doveri), trasformando così i soggetti in una sorta di cloni, privati delle caratteristiche individuali proprie di ognuno. Questa visione totalizzante, anche se può astrattamente sembrare giusta, tuttavia non è la visione ecclesiale, così come ce l'ha tramandata Cristo, e tutto sommato nemmeno appare corretta da un punto di vista "laico"; la Chiesa, infatti, riconosce le particolarità proprie di ciascuno e le valorizza, anche dal punto di vista giuridico, tenendo conto delle peculiarità rappresentate dalla scelta di uno stato di vita, scelta che avviene in modo assolutamente libero, e che tutti i fedeli, in quanto battezzati, sono in grado di compiere. Coloro che scelgono di non ricevere il sacramento dell'ordine non perdono in alcun modo la loro dignità di fedeli, ma sanno che hanno scelto di non svolgere determinate funzioni che sono riservate solamente a coloro che assumono lo stato clericale. In termini giuridici: il fedele non ordinato (laico o religioso che sia) parteciperà all'esercizio dei tria munera, ma in modo differente, e al tempo stesso particolare, rispetto a come li esercita colui che invece ha liberamente scelto di divenire un ministro ordinato. Il ministero che è chiamato a svolgere il fedele non ordinato, non inferiore a quello che svolge il fedele ordinato, è semplicemente di un altro ordine, si pone su di un altro piano, non necessariamente di minore importanza. Anche nell'ambito degli ordinamenti civili tale impostazione non pare essere errata: uguaglianza non significa riconoscere a tutti i medesimi diritti, ma riconoscere a ciascuna categoria di cittadini i diritti confacenti alla loro posizione nella società. Un minore, ad es., non può essere posto sul medesimo piano di un adulto, al contrario, gli dovranno essere riconosciute maggiori tutele giuridiche, proprio in virtù della sua posizione di inferiorità rispetto ad altri soggetti giuridici.

Ritornando ora al già ricordato principio di uguaglianza contenuto nel can. 208; esso comporta alcune conseguenze pratiche, in quanto l'uguaglianza tra i fedeli si concretizza in due ambiti: la dignità e l'azione. La dignità è ambito conseguente dal diritto divino e fa parte della condizione ontologica di ciascun fedele, mentre l'azione attiene in buona parte all'ambito del diritto umano, ed è quindi soggetta a possibili restrizioni 
dettate dal diritto umano. Possiamo perciò affermare che, a partire dalla definizione di uguaglianza contenuta nel can. 208, i vari diritti e doveri dei fedeli, trovano una regolamentazione più o meno ampia, a seconda che attengano al piano della dignità o a quello dell'azione.

Diritti e doveri dei fedeli non devono essere considerati allo stesso modo dei diritti e doveri riconosciuti ai cittadini degli Stati, come già ricordato, quanto piuttosto come mezzi indirizzati al conseguimento dei fini propri dell'ordinamento della Chiesa: la costituzione di una comunità ecclesiale giusta, che porti alla salvezza finale dell'individuo.

L'uguaglianza giuridica ${ }^{60}$ tra christifideles e i diritti-doveri che da essa traggono origine hanno, come vero contenuto, la dignità comune a tutti i fedeli, principio di diritto divino, che è diretta derivazione del concetto antropologico di persona imago Dei. Tale assoluta peculiarità del diritto canonico si riflette sui canoni contenuti nel Libro II del CIC, per cui, come affermato

per comprendere la naturalezza dei diritti fondamentali del fedele è necessario conoscere la dignità del fedele, non solo la dignità dell'uomo. Si esige siano affermati i dati naturali ed anche quelli soprannaturali. Le esigenze della dignità umana e le esigenze della filiazione divina e del sacerdozio comune. E si richiede inoltre di comprendere la dignità del fedele non in modo isolato, bensì nel seno della comunione con Dio e con gli altri fedeli nel Corpo di Cristo ${ }^{61}$.

${ }^{60}$ A proposito del principio di uguaglianza, un noto storico del diritto commentava: «L'uguaglianza giuridica di noi moderni, che è uguaglianza borghese, uguaglianza formale di persone concretamente disuguali, appare alla Chiesa come una mostruosità. Nei tribunali canonici di sempre, anche in quelli odierni, sarebbe inconcepibile la scritta "la legge è uguale per tutti", che noi laici amiamo ostentare con ingenua vanagloria nelle nostre aule giudiziarie, perché la legge canonica non può essere uguale per tutti se tutti non sono concretamente uguali, perché la legge canonica - che non è garanzia formale ma aiuto sostanziale - deve tener conto delle humanae fragilitates che ha di fronte e, per ordinarle adeguatamente, deve conformarsi ad esse, sacrificare logicità, sistematicità, unitarietà formali ed "elasticizzarsi" come una veste che vuole essere aderente ai diversi corpi sottostanti», P. Grossi, L'ordine giuridico medievale, RomaBari $2004^{11}, 120$.

${ }^{61}$ J. Otaduy, Derechos de los fieles (1980-2000), Fidelium Iura 10 (2000) 49-50; [la traduzione italiana è nostra n.d.r.]. 
La persona, che per mezzo del Battesimo viene incorporata alla realtà ecclesiale, si trova in una condizione differente da quella in cui si trova un qualsiasi consociato di qualsivoglia ordinamento giuridico; il fedele è immerso in una realtà assolutamente unica, la Chiesa, la quale, dal punto di vista istituzionale rappresenta

tutto ciò che nella comunione trascende le singole persone. Tuttavia, in quei rapporti che si instaurano con la Chiesa istituzionale non va dimenticato che il vero protagonista è la persona. Pertanto la nuova situazione giuridica in cui viene a trovarsi la persona grazie al battesimo consiste essenzialmente in un vincolo comunionale che lo lega con tutti i suoi fratelli nella Chiesa: è nei loro riguardi che esistono i diritti e i doveri battesimali, i quali ovviamente anche possiedono pieno valore nel rapporto con la Chiesa quale istituzione, essendo questo rapporto finalizzato alle stesse persone ${ }^{62}$.

Nella Chiesa, e di conseguenza nel suo sistema normativo, diritti ed obblighi assumono dunque un significato del tutto particolare: essi devono essere visti come strumenti, piuttosto che come mezzi giuridici per la rivendicazione di un ambito di autonomia da parte del fedele-consociato. Diritti ed obblighi sono gli strumenti attraverso i quali si garantisce ai fedeli quel minimo di indirizzo normativo per lo svolgimento, all'interno della realtà ecclesiale, della loro missione, che vede nella salus animarum il suo fine ultimo. Se invece si volessero leggere diritti e doveri allo stesso modo di quelli che sono i diritti e doveri dell'uomo, o del cittadino, riconosciuti da un qualsiasi ordinamento giuridico civile, si riscontrerebbe in essi solamente un limite alla libertà di ogni singolo fedele-consociato.

Il fedele, invece, deve poter ritrovare in questi canoni le linee guida per il proprio operare all'interno della Chiesa; tali diritti ed obblighi, sono solo una parte di quello che potremmo denominare statuto del fedele, in quanto disciplinano alcuni aspetti del vivere ecclesiale, senza nulla togliere a tutto quanto fa parte dell'essere persona, e persona battezzata, in senso generale.

${ }^{62}$ C.J. ERrÁzUriz M., Riflessioni sul rapporto tra battesimo e situazione giuridicocanonica della persona, Fidelium Iura 6 (1996) 148-149. 
A tal proposito possiamo pensare alla rilevanza che assumono i carismi personali nella definizione dello statuto del fedele all'interno dell'ordinamento canonico; carismi che purtroppo non hanno trovato un loro esplicito riferimento nelle norme codiciali riguardanti i fedeli. Basti questo esempio per mostrare come non si possa valutare efficacemente lo statuto del fedele solamente avendo presente il dato normativo positivo, per quanto esso possa essere utilizzato per delimitare i confini entro cui si deve ordinatamente sviluppare tale statuto. È invece necessario tener presenti molteplici aspetti, tutti ugualmente importanti e tutti compenetrantisi tra loro. Quando si riconosce ai fedeli un certo ambito di autonomia e di libertà, questi non debbono essere letti come si leggerebbero in un qualsiasi ordinamento positivo, per cui nessuno può intromettersi nella sfera dei diritti propri del singolo (salvo poi venire totalmente privata tale sfera, di fronte ad interessi pubblici “superiori”), ma al contrario, l'autorità interviene per meglio sviluppare tali ambiti, per far fruttare, ad esempio, i doni dello Spirito che altrimenti rischierebbero di rimanere incompresi.

In un sistema come quello canonico, il fedele è obbligato non solamente a livello giuridico dai canoni di cui si sta qui discutendo, avendo egli obblighi di tipo ad esempio morale che a quelli giuridici si affiancano e che non sempre possono essere canonizzati, ma che non per questo non sono fonte per ogni battezzato di diritti ed obblighi parimenti importanti per conseguire la salvezza. Proprio in virtù di tale caratteristica, che fa parte della condizione stessa del battezzato, non si possono né comprendere né applicare i canoni in oggetto attenendosi a criteri di tipo esclusivamente positivistico. Il fedele, nell'accostarsi a questi canoni, deve anzitutto chiedersi, in relazione ad una situazione concreta di applicazione, in che cosa consista il suo diritto o il suo obbligo, visti non come rivendicazione o costrizione, ma come strumento per compiere un altro decisivo passo verso la salvezza.

In questa prospettiva, è indispensabile che il fedele si interroghi, di volta in volta, per capire quale sia la cosa giusta da fare o da non fare, in un'analisi che lo porterà a trovare sempre nuove prospettive interpretative della norma canonica, e che renderanno queste norme sempre 
più utili per la sua salvezza. Con il Battesimo, non si riceve la cittadinanza di uno Stato, acquisita la quale si possiede un bagaglio di diritti e doveri; con il Battesimo si diviene christifideles, per cui, assunta tale condizione nuova, si è chiamati ad un rinnovamento totale che passa anche attraverso un rinnovamento della propria dimensione giuridica ${ }^{63}$.

L'attenzione di molti commentatori, circa i canoni riguardanti obblighi e diritti dei fedeli, si è soffermata sulla mancata codificazione di appositi strumenti volti a rendere effettiva la tutela dei diritti ivi affermati; ad esempio il mancato inserimento di norme che disciplinino il ricorso amministrativo. Pur senza sminuire l'importanza che tali strumenti possiedono nell'ambito di un ordinamento giuridico, ci sembra non siano così indispensabili, nell'ambito canonico, per la tutela dei diritti dei fedeli, dal momento che tali diritti possiedono una forza obbligante loro propria che deriva dall'essere diritti di origine superiore, e pure dal fatto che è prevista la possibilità di segnalare, a chi deputato a compiti di governo superiori, quelle che sono le esigenze del fedele e talvolta della Chiesa stessa, come indicato nel can. 212 § 3: «In modo proporzionato alla scienza, alla competenza e al prestigio di cui godono, essi hanno il diritto, e anzi talvolta anche il dovere, di manifestare ai sacri Pastori il loro pensiero su ciò che riguarda il bene della Chiesa; e di renderlo noto agli altri fedeli, salva restando l'integrità della fede e dei costumi e il rispetto verso i Pastori, tenendo inoltre presente l'utilità comune e la dignità della persona».

I canoni presi in esame, anche a livello terminologico, parlano di cooperazione, di impegno personale, di responsabilità, di comunione, quasi a sottolineare come il fedele sia chiamato a cooperare al corretto

\footnotetext{
${ }^{63}$ Il fedele dovrebbe poter affermare, di fronte ai mezzi che la Chiesa gli offre per conseguire la salvezza, come a suo tempo sant'Agostino: «È tanta la bellezza della giustizia, tanto l'incanto della luce eterna, cioè della immutabile verità e sapienza che, anche nell'ipotesi che si potesse rimanere in essa per lo spazio di un sol giorno, per questo stesso motivo si dovrebbero disprezzare molto giustamente innumerevoli anni di questa vita, pieni di delizie e abbondanza di beni temporali. Infatti non è stato detto erroneamente o con scarso sentimento: Un solo giorno nei tuoi atrii è migliore di mille giorni (Sal 83,11)», SANT'Agostino, De libero arbitrio, 3.25.77. La traduzione italiana è in www.augustinus.it/italiano/libero_arbitrio/.
} 
sviluppo della società (ecclesiale) in cui vive, e pure per la effettiva applicazione dei principi elencati nei canoni relativi a diritti ed obblighi di tutti i fedeli.

\section{Diritti ed obblighi dei fedeli laici}

Posto che i canoni riguardanti doveri e diritti dei fedeli, riguardano anche i fedeli laici ${ }^{64}$, il CIC attualmente vigente dedica uno specifico titolo (il II) ai doveri e diritti dei fedeli laici; tali doveri e diritti, pertanto, si sommano a quelli già contenuti nel Titolo I del Libro II. Il can. 224, nello specificare che ai fedeli laici sono riconosciuti tutti i diritti ed obblighi propri e comuni a tutti i fedeli, a prescindere dal loro personale stato, è indicativo di ciò che siamo andati sin qui osservando, ovvero che tali canoni hanno come presupposto la tutela dell'uomo e dunque vanno riconosciuti a tutti i fedeli, a prescindere dalle loro personali scelte di vita, che li portano ad abbracciare un determinato stato giuridico.

Tuttavia il riconoscimento della fondamentale dignità ed uguaglianza di tutti i fedeli, non cancella la costituzione gerarchica propria della Chiesa, per cui, a seconda della scelta personale di ognuno, sono attribuiti più specifici diritti e doveri, caratterizzanti tale personale stato. È stato in particolare osservato che vi sono quattro grandi principi che caratterizzano lo statuto giuridico dei fedeli laici: la vocazione universale alla santità, la libertà ed autonomia nell'ambito temporale, la libertà di apostolato e la capacità di svolgere determinati uffici ed incarichi ecclesiastici ${ }^{65}$.

Vi è però, tra $\mathrm{i}$ canoni riguardanti i fedeli laici, una norma che appare a nostro avviso come assolutamente prevalente rispetto alle altre, in quanto caratterizza in maniera del tutto peculiare i fedeli che scelgono di condurre la propria vita rimanendo nello stato laicale: è il dettato del can. $225 \S 2$, in base al quale: «Sono tenuti anche al dovere specifico, ciascuno secondo la propria condizione, di animare e perfezionare

${ }^{64}$ Un'ampia bibliografia sul tema dei diritti dei fedeli laici è contenuta in: C.-M. FABris, Il Popolo di Dio. I. I diritti dei fedeli. I diritti dei fedeli laici, Ephemerides Iuris Canonici 53 (2013) 205-239.

65 J. ForNÉs, La condición jurídica del laico en la Iglesia, Ius Canonicum 51 (1986) 35-61. 
l'ordine delle realtà temporali con lo spirito evangelico, e in tal modo rendere testimonianza a Cristo particolarmente nel trattare tali realtà e nell'esercizio dei compiti secolari». Tale canone descrive infatti la realtà di vita del laico, che è rappresentata primariamente (dovere specifico) dal vivere la realtà temporale in modo tale da rendere testimonianza della propria fede cristiana.

Questa è senza dubbio la caratterizzazione più forte del fedele laico, quella che meglio lo definisce dal punto di vista giuridico, differenziandolo in maniera netta dagli altri fedeli nella Chiesa; il laico, senza distinzioni tra uomo e donna (dato di una certa rilevanza) è chiamato a vivere nel secolo ed a portare in esso la propria testimonianza di vita cristiana. Il laico deve vivere il proprio particolare statuto giuridico nella vita di tutti i giorni, ove svolge un ruolo assolutamente fondamentale che non si deve limitare solamente ad animare le realtà temporali, ma addirittura a perfezionarle alla luce dello spirito evangelico del quale egli è insostituibile testimone ${ }^{66}$.

Il laico in sostanza deve fare il laico, deve vivere la propria vita nel mondo facendosi portatore del messaggio evangelico secondo il proprio peculiare stato di vita, che non è il medesimo stato dei chierici e nemmeno quello dei religiosi. Tale avvertimento vale ovviamente anche in senso inverso: chierici e religiosi dovranno vivere secondo il loro peculiare stato di vita, senza pretendere di svolgere il ruolo che è proprio dei laici. Non si tratta ovviamente di una lotta di rivendicazione di spazi propri, ma di riconoscere che ogni scelta di vita ha delle conseguenze pratiche sulla persona che devono essere pienamente ed appropriatamente vissute. Inoltre tali scelte caratterizzano ciascun fedele anche dal punto di vista giuridico, non in quanto la scelta dello

\footnotetext{
${ }^{66}$ Dell'indole secolare, quale caratteristica propria e peculiare dei fedeli laici, parla diffusamente la Esortazione Ap. Christifideles laici (30 dicembre 1988) al n. 15. In tale fondamentale documento, si affronta la figura del fedele laico sotto molteplici punti di vista; in particolare si ricordano le molteplici funzioni che il laico è chiamato a compiere nella Chiesa (n. 23 ss.) e le peculiarità proprie della vocazione laicale (n. 55 ss.). Tale documento costituisce il punto di arrivo delle riflessioni post-conciliari sul fedele laico, ed al tempo stesso il punto di partenza per una giusta considerazione del laico nella Chiesa oggi.
} 
stato di vita comporti una differenziazione sul piano giuridico con attribuzione di maggiori o minori diritti e doveri, ma in quanto la scelta personale e libera operata da ciascuno ha delle ripercussioni necessarie anche sul piano delle attribuzioni da parte dell'ordinamento ${ }^{67}$; d'altra parte la vera uguaglianza come (dovrebbe essere) noto, non consiste nel dare tutto a tutti, ma a ciascuno il suo.

All'interno dello stato laicale, poi, esistono ulteriori specificazioni che vengono alla luce man mano che si approfondisce la particolare situazione di ciascun individuo; il can. 226 contiene in tal senso due chiare indicazioni: i laici possono essere sposati, ed ecco che i loro diritti e doveri assumono delle connotazioni maggiormente definite; i coniugi possono divenire genitori, ed ancora il loro personale statuto subirà delle necessarie specificazioni ${ }^{68}$. Non si tratta, come evidente, di discriminazioni nei confronti degli altri fedeli, ma semplicemente di un più pieno riconoscimento delle particolarità proprie di ciascun soggetto, che non fanno altro che tutelare e definire in maniera sempre più precisa ed accurata il valore di riferimento: l'uomo, l'uomo che sceglie di vivere "nel mondo", l'uomo che sente la vocazione di divenire ministro ordinato, l'uomo che sceglie di vivere più pienamente i consigli evangelici, l'uomo che sceglie di divenire sposo, l'uomo che sceglie di generare dei figli. Mano a mano che si scende nello specifico, non

${ }^{67}$ A proposito di una supposta disuguaglianza esistente tra i laici ed i chierici, è stato acutamente osservato che, in base alla conditio libertatis riconosciuta ad ogni fedele in virtù del battesimo, i laici avranno addirittura un più ampio spazio di libertà personale «poiché soltanto i laici non hanno assunto nei confronti della Chiesa, in quanto struttura societaria, ulteriori impegni atti a modificare l'originario stato di libertà e di autonomia contenuto nel Battesimo», J. I. ARRIETA, Libertà fondamentali e libertà fondamentale. Considerazioni attorno alla diversa posizione dei fedeli nella partecipazione alla missione della Chiesa, in: AA. Vv., I diritti fondamentali del fedele. A vent'anni dalla promulgazione del Codice, Città del Vaticano, 2004, 199-208, in partic. 205.

${ }^{68}$ Le ulteriori specificazioni circa lo statuto giuridico personale dei laici, in relazione alla libera scelta dello stato di vita, ha delle concrete e pratiche ricadute giuridiche, in riferimento all'esercizio del munus docendi. Basta osservare le norme contenute nel Libro III dell'attuale CIC, per rendersi conto che, anche a livello terminologico, alcuni canoni riprendono quelli contenuti nel Libro II. I richiami al ruolo che i laici sono chiamati a svolgere in tale ambito sono spesso molto forti come accade, ad esempio, nel caso dei genitori in relazione alla educazione dei figli. 
si perde ciò che è riconosciuto in senso più generale, ma viene invece meglio precisato ciò che è proprio di ciascuna condizione: ecco perché gli obblighi e i diritti di tutti i fedeli si pongono come le norme assolutamente fondamentali, esse sono la base ed il cardine di tutte le altre e poggiano sul riconoscimento del valore proprio della persona umana creata ad immagine di Dio.

Le norme che riguardano più specificamente obblighi e diritti dei fedeli laici, sono da comprendersi pur sempre alla luce del dettato del can. 223, il quale costituisce un chiaro indizio dello spirito con cui si devono sviluppare le relazioni tra fedeli appartenenti ai diversi stati: il fedele, è chiamato a «tener conto del bene comune della Chiesa, come pure dei diritti altrui e dei propri doveri nei confronti degli altri». Il fedele non deve solamente tenere o non tenere determinate condotte in quanto proprie del suo stato, deve impegnarsi in prima persona nella realizzazione di un ordine di giustizia, a volte sacrificando anche quelle che possono essere le sue legittime aspirazioni, nella convinzione che lo si fa in vista di un traguardo più alto di quelli che possono essere i banali traguardi quotidiani. A volte sacrificare una propria aspirazione, pure legittima, può servire a conseguire un bene ed una giustizia maggiori rispetto a quella materiale che si potrebbe ottenere attraverso un'azione giudiziale di un certo tipo, o alla rivendicazione di un diritto concreto. Se così non fosse, in cosa si distinguerebbe colui che ha ricevuto una vita nuova nel Battesimo in Cristo, da coloro i quali non sono ancora giunti ad abbracciare la via della salvezza? Il messaggio evangelico è chiaro, prima di tutto vi è l'impegno personale e quotidiano, attraverso il quale si giunge alla salvezza; si deve puntare ad una giustizia più grande di quella che può essere concessa in modo imperfetto in un tribunale terreno. Il laico deve collaborare con il chierico ed il religioso alla edificazione della Chiesa di Cristo, e tale collaborazione deve essere reciproca e rispettosa delle istanze proprie di ciascuno, senza che vengano avanzate pretese indebite nei confronti degli altri. 


\section{The faithfuls' rights as expression of values}

This article offers a broad analysis on the theme relevant to the canonical subject "The People of God". It provides particularly insight into the theme of the rights of the faithful within the ecclesiastical framework, with special emphasis on the rights of the lay faithful. The discussion regarding the recognition of the rights of the faithful and those of the laity, has indeed characterized canonical scholarship since the nineteenth century, influencing also the innovations of the second Vatican Council. All the theme of the rights is focused in an optics of "rights as values". The author provides also a panoramic overview of the relevant canonical literature, making reference to the innovations introduced in the course of time by subsequent juridical reforms.

Parole chiave: fedeli, laici, diritti dei fedeli, obblighi dei fedeli

KEY wORDS: Christ's faithful, lay peple, rights of Christ's faithful, obbligations of Christ's faithful 